\title{
Minimum Spanning Tree of City to City Road Network in Nigeria
}

\author{
Effanga, E.O. \& Edeke, Uwe. E. \\ Department Of Statistics, University Of Calabar, Nigeria
}

\begin{abstract}
This paper deals with the construction of minimum spanning tree of city - to - city network in Nigeria. Nodes in our network are the 36 state capitals in Nigeria and the FCT, and the arcs are the proposed major roads that link the state capitals. The distances between states are computed using the Lad/Long converter software which makes use of the latitude and longitude of each state capital. We employed Prim's algorithm to determine the minimum spanning tree with Yenagoa as the starting point. The result gives a total distance of 5, $128.5 \mathrm{~km}$.
\end{abstract}

Keywords: Spanning tree, Network, Prim's algorithm, Latitude and Longitude

\section{Introduction}

A lot of researches have been done on designing optimal network for purposes of distribution, communication, machine scheduling, gas and pipelines, etc. The tool commonly used for the optimal design of such networks is the Minimal Spanning Tree. A Minimal Spanning Tree problem is one of the most fundamental and intensively studied problems in network optimization problem with many theoretical and practical applications (Ahuja, et al, 1993), (Taha, 2006), (Winston, 2004), (Dippon, 1999), (Seth, 2002), (Nahla, 2011), (Mares, 2008). (Rothfard, 1970) used minimum spanning tree to design optimal offshore natural gas pipeline systems

A number of algorithms exist for the determination of Minimum Spanning Tree; these include Kruskal algorithm, Prim's algorithm and Boruvka algorithm, (Agarval, 2010). The Prim's algorithm was developed in 1957 by a computer scientist, Robert C. Prim and rediscovered by Edsger Diijkstra in the same year. For this reason the algorithm is sometimes known as DJP algorithm (Wilkipedia, 2010). An optimal design of natural gas pipeline of Amaco East Cross field gas pipeline project in Alberta - Canada with a total distance of $66 \mathrm{~km}$ was reduced to $49.9 \mathrm{~km}$ by using hamster program software. Steiner minimum spanning tree algorithm was later used to reduce the distance to $48.84 \mathrm{~km}$ (Dott, 1997).

A study on the optimal design of oil pipeline network for the South Gabon oil field having 33 nodes and 129 possible arcs reduces the total distance of 188.2 miles to 156.2 miles using Prim's algorithm (Brimberg et al, 2003). (Donkor et al, 2011) used Prim's algorithm to determine the Minimum Spanning Tree of length $712 \mathrm{~km}$ of the West African gas pipeline from Nigeria through Benin and Togo to Ghana.

In this paper, we construct a Minimum Spanning Tree covering 36 state capitals and FCT, namely: Yenagoa, Port - Harcourt, Oweri, Asaba, Awka, Umuhia, Uyo, Calabar, Ababkiliki, Enugu, Lokoja, Lafia, Makurdi, Jalingo, Yola, Maiduguri, Damaturu, Gombe, Bauchi, Jos, Kaduna, Minna, Ilorin, Oshogbo, Adoekiti, Akure, Benin city, Ikeja, Abeokuta, Ibadan, Berrin - Kebbi, Sokoto, Gusau, Katsina, Kano, Dutse and Abuja.

\section{City - to - City road Network in Nigeria}

\section{Methodology}

A network is a collection of points (nodes) linked by arcs (branches). Let $\mathrm{N}$ be a set of finite number of nodes, and $\mathrm{A}$ be a set of arcs linking the nodes. Then a network is defined by the pair $(\mathrm{N}, \mathrm{A})$. In a network, the arc linking two distinct nodes $i$ and $j$ is denoted by $(i, j)$. In every network, there is a flow of some type along its arcs. For instance, in road network, the flows are the vehicle; in communication network, the flows are the messages along the wires; in pipeline network, the flows are oil products, etc. If flows are allowed in only one direction along an arc, the arc is said to be directed or oriented. If all the arcs in a network are directed, then the network is called a directed network, otherwise it is undirected (Hillier \& Lieberman, 2001).

A sequence of arcs linking two distinct nodes forms a path in a network. A path forms a loop or cycle in a network if it connects a node to itself. Two nodes in a network are connected if there is at least one path linking them. When all the nodes in a network are linked by at least one path, the network is said to be connected (Taha, 2006).In this paper, a city - to - city road network in Nigeria is constructed first. Then Prim's algorithm is employed to obtain a minimum spanning tree. Figure 1 shows City - to - City road network in Nigeria, while Fig. 2 shows minimum spanning tree of the network.

\section{Tree}

A tree is any subset of a network not containing a loop. Given a set of nodes N, a tree can be grown by linking any two nodes by an arc, and subsequently adding new arc in such a way that it links a node already linked to other nodes to a new node not previously linked to any other node. When nodes are linked this way, the problem of creating a chain is avoided and the number of nodes linked will be 1 greater than the number of arcs. (Taha, 2002) A tree is spanning tree if all the nodes in a network are linked and are connected. Every spanning tree has exactly (m -1) arcs in a network of m nodes, since this is the minimum number of arcs needed to have a connected network and the maximum number possible without having a chain.

\section{The Minimal Spanning Tree problem}

A spanning tree is a group of $(\mathrm{m}-1)$ arcs that links all the m nodes of the network and contains no chain. A spanning tree of minimum length in a network is a minimum spanning tree. The minimal spanning tree problem found its applications in the creation of a network of paved roads that links several rural towns, where the road between two towns may pass through one or more other towns.

The Minimal Spanning Tree algorithm (The Prim's Algorithm)

The steps are as follows:

Step 0: set $C=\varnothing$ and $C^{\prime}=\{1,2, \cdots, m\}$ 
Step 1: Start with any node, say node $i$, in $C_{0}^{\prime}$ and connect it to node $\mathrm{j}$ that is closest to node $i$. Set $C=\{i, j\}$ and $C^{\prime}=\{1,2, \cdots, m\} \backslash\{i, j\}$.

Step 2: Now choose node 1 in $C^{\prime}$ that is closest to some node $k$ in $C$. Then connects node $k$ to node 1 . Set $C=\{i, j$, $\mathrm{k}\}$ and $\mathrm{C}^{\prime}=\mathrm{C}^{\prime} \backslash \mathrm{C}$

Step 3: Repeat step 2 until $C^{\prime}=\varnothing$. Ties for the closest node and arc to be included in the minimum spanning tree may be broken arbitrarily. (Hillier and Lieberman, 2001).

\section{Method of Data collection}

The data used for this study is generated using Lad/Long converter software which makes use of latitude and longitude of cities in Nigeria to determine the distances between cities. Table 1 shows latitude and longitude of each state capital, while Table 2 shows city - to - city distances.

\section{City-to-City Road Network in Nigeria.}

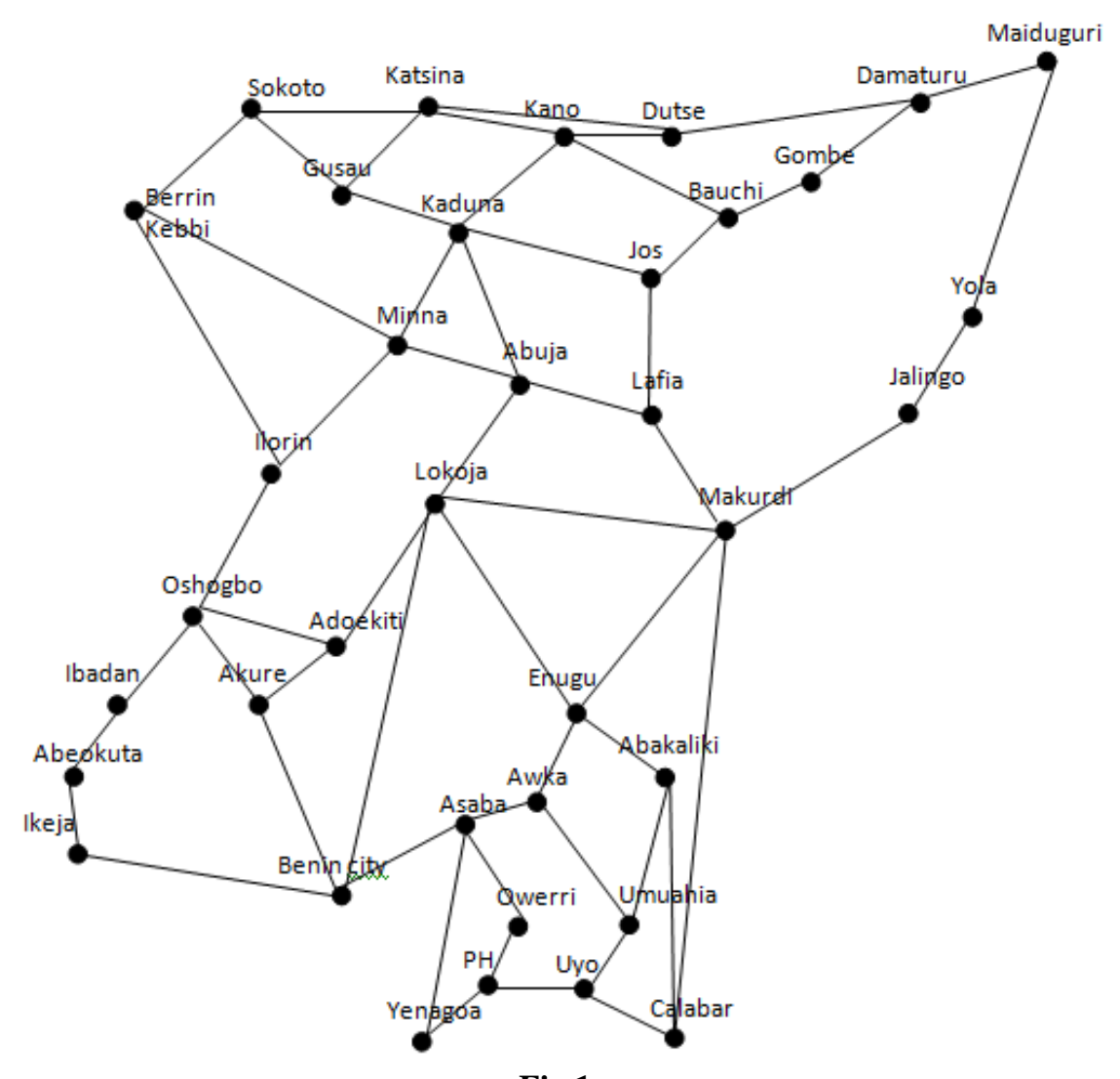

Fig.1

Table 1: Latitudes and Longitudes of State capitals in Nigeria

\begin{tabular}{|c|c|c|c|c|c|c|c|}
\hline $\mathrm{S} / \mathrm{N}$ & STATE CAPITALS & LATITUDE & LONGITUDE & $\mathrm{S} / \mathrm{N}$ & STATE CAPITALS & LATITUDE & LONGITUDE \\
\hline 1 & Ikeja & $6.5833^{\circ} \mathrm{N}$ & $3.3333^{\circ} \mathrm{E}$ & 23 & Lafia & $8.4917^{\circ} \mathrm{N}$ & $8.5167^{\circ} \mathrm{E}$ \\
\hline 2 & Abeokuta & $7.1608^{\circ} \mathrm{N}$ & $3.3483^{\circ} \mathrm{E}$ & 24 & Jos & $9.9333^{\circ} \mathrm{N}$ & $8.8833^{\circ} \mathrm{E}$ \\
\hline 3 & Ibadan & $7.3964^{\circ} \mathrm{N}$ & $3.9167^{\circ} \mathrm{E}$ & 25 & Bauchi & $10.5000^{\circ} \mathrm{N}$ & $10.0000^{\circ} \mathrm{E}$ \\
\hline 4 & Oshogbo & $7.7660^{\circ} \mathrm{N}$ & $4.5667^{\circ} \mathrm{E}$ & 26 & Gombe & $10.2500^{\circ} \mathrm{N}$ & $11.1667^{\circ} \mathrm{E}$ \\
\hline 5 & Ado-Ekiti & $7.6211^{\circ} \mathrm{N}$ & $5.2214^{\circ} \mathrm{E}$ & 27 & Jalingo & $8.9000^{\circ} \mathrm{N}$ & $11.3667^{\circ} \mathrm{E}$ \\
\hline 6 & Akure & $7.2500^{\circ} \mathrm{N}$ & $5.1950^{\circ} \mathrm{E}$ & 28 & Yola & $9.2300^{\circ} \mathrm{N}$ & $12.4600^{\circ} \mathrm{E}$ \\
\hline 7 & Benin City & $6.3176^{\circ} \mathrm{N}$ & $5.6145^{\circ} \mathrm{E}$ & 29 & Maiduguri & $11.8333^{\circ} \mathrm{N}$ & $13.1500^{\circ} \mathrm{E}$ \\
\hline 8 & Asaba & $6.1978^{\circ} \mathrm{N}$ & $6.7285^{\circ} \mathrm{E}$ & 30 & Damaturu & $11.7444^{\circ} \mathrm{N}$ & $11.9611^{\circ} \mathrm{E}$ \\
\hline 9 & Awka & $5.0000^{\circ} \mathrm{N}$ & $7.8333^{\circ} \mathrm{E}$ & 31 & Dutse & $11.7011^{\circ} \mathrm{N}$ & $9.3419^{\circ} \mathrm{E}$ \\
\hline 10 & Enugu & $6.4527^{\circ} \mathrm{N}$ & $7.5103^{\circ} \mathrm{E}$ & 32 & Kano & $12.0000^{\circ} \mathrm{N}$ & $8.5167^{\circ} \mathrm{E}$ \\
\hline 11 & Abakaliki & $6.3333^{\circ} \mathrm{N}$ & $8.1000^{\circ} \mathrm{E}$ & 33 & Katsina & $12.2500^{\circ} \mathrm{N}$ & $7.5000^{\circ} \mathrm{E}$ \\
\hline 12 & Umuahia & $5.5333^{\circ} \mathrm{N}$ & $7.4833^{\circ} \mathrm{E}$ & 34 & Gusau & $12.1500^{\circ} \mathrm{N}$ & $6.6667^{\circ} \mathrm{E}$ \\
\hline 13 & Calabar & $4.7500^{\circ} \mathrm{N}$ & $8.3250^{\circ} \mathrm{E}$ & 35 & Sokoto & $13.0667^{\circ} \mathrm{N}$ & $5.2333^{\circ} \mathrm{E}$ \\
\hline 14 & Uyo & $5.0500^{\circ} \mathrm{N}$ & $7.9333^{\circ} \mathrm{E}$ & 36 & Berrin - Kebbi & $11.5000^{\circ} \mathrm{N}$ & $4.0000^{\circ} \mathrm{E}$ \\
\hline 15 & Owerri & $5.4850^{\circ} \mathrm{N}$ & $7.0350^{\circ} \mathrm{E}$ & 37 & Kaduna & $10.5167^{\circ} \mathrm{N}$ & $7.4333^{\circ} \mathrm{E}$ \\
\hline 16 & Port - Harcourt & $6.3176^{\circ} \mathrm{N}$ & $7.0000^{\circ} \mathrm{E}$ & & & & \\
\hline 17 & Yenagoa & $4.7500^{\circ} \mathrm{N}$ & $6.3333^{\circ} \mathrm{E}$ & & & & \\
\hline 18 & Ilorin & $8.5000^{\circ} \mathrm{N}$ & $4.5500^{\circ} \mathrm{E}$ & & & & \\
\hline 19 & Minna & $9.6139^{\circ} \mathrm{N}$ & $6.5569^{\circ} \mathrm{E}$ & & & & \\
\hline 20 & Abuja & $9.0667^{\circ} \mathrm{N}$ & $7.4833^{\circ} \mathrm{E}$ & & & & \\
\hline 21 & Lokoja & $7.8167^{\circ} \mathrm{N}$ & $6.7500^{\circ} \mathrm{E}$ & & & & \\
\hline 22 & Makurdi & $7.7306^{\circ} \mathrm{N}$ & $8.5361^{\circ} \mathrm{E}$ & & & & \\
\hline
\end{tabular}


Minimum Spanning Tree Of City To City Road Network In Nigeria

Table 2: City - to - City Road Network showing Distances in KM

\begin{tabular}{|c|c|c|c|c|c|c|c|c|c|c|c|c|c|c|}
\hline CITY & 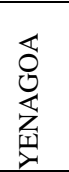 & $\stackrel{\pi}{\Omega}$ & $\begin{array}{l}\vec{a} \\
\frac{\pi}{\pi} \\
\frac{\pi}{3}\end{array}$ & 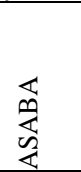 & $\frac{\mathbb{s}}{3}$ & 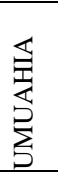 & 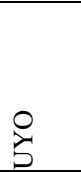 & 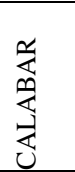 & 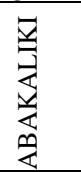 & 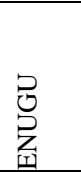 & $\begin{array}{l}\overleftarrow{3} \\
0 \\
0 \\
0\end{array}$ & 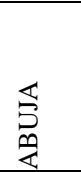 & 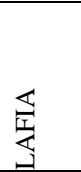 & 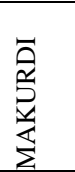 \\
\hline YENAGOA & & 80.3 & & 136.6 & & & & & & & & & & \\
\hline $\mathrm{PH}$ & 80.3 & & 81.3 & & & & 108.6 & & & & & & & \\
\hline OWERRI & & & & 86.2 & & & & & & & & & & \\
\hline ASABA & & & & & 180.7 & & & & & & & & & \\
\hline AWKA & & & & 180.7 & & 70.8 & & & & 165.3 & & & & \\
\hline UMUAHIA & & & & & 70.8 & & 73.2 & & & & & & & \\
\hline UYO & & 108.6 & & & & 73.2 & & 44.9 & & & & & & \\
\hline CALABAR & & & & & & & 44.9 & & 115.7 & & & & & 309.9 \\
\hline ABAKALIKI & & & & & & & & 115.7 & & 85.5 & & & & \\
\hline ENUGU & & & & & 165.3 & & & 85.5 & 85.5 & & 173.2 & & & 181.6 \\
\hline LOKOJA & & & & & & & & & & 173.2 & & 160.6 & & 196.9 \\
\hline ABUJA & & & & & & & & & & & 160.6 & & 130.2 & \\
\hline LAFIA & & & & & & & & & & & & 130.2 & & 84.6 \\
\hline MAKURDI & & & & & & & & 309.9 & & 181.6 & 196.9 & & 84.6 & \\
\hline JALINGO & & & & & & & & & & & & & & 337.3 \\
\hline YOLA & & & & & & & & & & & & & & \\
\hline MAIDUGURY & & & & & & & & & & & & & & \\
\hline DAMATURU & & & & & & & & & & & & & & \\
\hline GOMBE & & & & & & & & & & & & & & \\
\hline BAUCHI & & & & & & & & & & & & & & \\
\hline JOS & & & & & & & & & & & & & 165.2 & \\
\hline KADUNA & & & & & & & & & & & & 161.2 & & \\
\hline MINNA & & & & & & & & & & & & 118.4 & & \\
\hline ILORIN & & & & & & & & & & & & & & \\
\hline OSHOGBO & & & & & & & & & & & & & & \\
\hline ADOEKITI & & & & & & & & & & & 381.8 & & & \\
\hline AKURE & & & & & & & & & & & & & & \\
\hline BENIN CITY & & & & 123.8 & & & & & & & 208.4 & & & \\
\hline IKEJA & & & & & & & & & & & & & & \\
\hline ABEOKUTA & & & & & & & & & & & & & & \\
\hline IBADAN & & & & & & & & & & & & & & \\
\hline BERIN KEBI & & & & & & & & & & & & & & \\
\hline SOKOTO & & & & & & & & & & & & & & \\
\hline GUSAU & & & & & & & & & & & & & & \\
\hline KATSINA & & & & & & & & & & & & & & \\
\hline KANO & & & & & & & & & & & & & & \\
\hline DUTSE & & & & & & & & & & & & & & \\
\hline
\end{tabular}

\begin{tabular}{|c|c|c|c|c|c|c|c|c|c|c|c|c|c|}
\hline CITY & \begin{tabular}{l}
$O$ \\
\hdashline \\
$Z$ \\
$Z$
\end{tabular} & 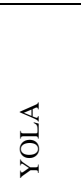 & 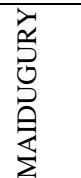 & 总 & $\sum_{0}^{\text {땡 }}$ & 敢 & $\tilde{\rho}$ & 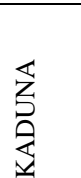 & 売 & Z & $\begin{array}{l}0 \\
0 \\
0 \\
0 \\
0 \\
0 \\
0\end{array}$ & $\begin{array}{l}\bar{\Xi} \\
\frac{1}{8} \\
8 \\
8\end{array}$ & 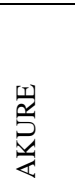 \\
\hline \multicolumn{14}{|l|}{ YENAGOA } \\
\hline \multicolumn{14}{|l|}{$\mathrm{PH}$} \\
\hline \multicolumn{14}{|l|}{ OWERRI } \\
\hline \multicolumn{14}{|l|}{ ASABA } \\
\hline \multicolumn{14}{|l|}{ AWKA } \\
\hline \multicolumn{14}{|l|}{ UMUAHIA } \\
\hline \multicolumn{14}{|l|}{ UYO } \\
\hline \multicolumn{14}{|l|}{ CALABAR } \\
\hline \multicolumn{14}{|l|}{ ABAKALIKI } \\
\hline \multicolumn{14}{|l|}{ ENUGU } \\
\hline LOKOJA & & & & & & & & & & & & 381.8 & \\
\hline ABUJA & & & & & & & & 161.2 & 118.4 & & & & \\
\hline LAFIA & & & & & & & 165.2 & & & & & & \\
\hline MAKURDI & 337.3 & & & & & & & & & & & & \\
\hline JALINGO & & 125.5 & & & & & & & & & & & \\
\hline YOLA & 125.5 & & 298.9 & & & & & & & & & & \\
\hline MAIDUGURY & & 298.9 & & 129.7 & & & & & & & & & \\
\hline DAMATURU & & & 129.7 & & 187.3 & & & & & & & & \\
\hline GOMBE & & & & & & 130.5 & & & & & & & \\
\hline BAUCHI & & & & & 130.5 & & 137.4 & & & & & & \\
\hline JOS & & & & & & 137.4 & & 171.3 & & & & & \\
\hline KADUNA & & & & & & & 171.3 & & 138.7 & & & & \\
\hline MINNA & & & & & & & & 138.7 & & 252.6 & & & \\
\hline ILORIN & & & & & & & & & 252.6 & & 81.5 & & \\
\hline OSHOGBO & & & & & & & & & & 81.5 & & 73.9 & 89.9 \\
\hline ADOEKITI & & & & & & & & & & & 73.9 & & \\
\hline AKURE & & & & & & & & & & & 89.9 & 203.8 & \\
\hline BENIN CITY & & & & & & & & & & & & & 113.5 \\
\hline \multicolumn{14}{|l|}{ IKEJA } \\
\hline \multicolumn{14}{|l|}{ ABEOKUTA } \\
\hline IBADAN & & & & & & & & & & 141.1 & 82.6 & & \\
\hline BERIN KEBI & & & & & & & & & 349.2 & 338.8 & & & \\
\hline \multicolumn{14}{|l|}{ SOKOTO } \\
\hline GUSAU & & & & & & & & 199.8 & & & & & \\
\hline \multicolumn{14}{|l|}{ KATSINA } \\
\hline KANO & & & & & & 232.2 & & 282.7 & & & & & \\
\hline DUTSE & & & & 285 & & & & & & & & & \\
\hline
\end{tabular}


Minimum Spanning Tree Of City To City Road Network In Nigeria

\begin{tabular}{|c|c|c|c|c|c|c|c|c|c|c|}
\hline CITY & 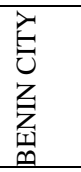 & 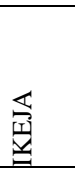 & 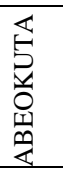 & 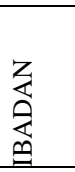 & 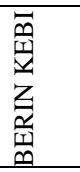 & 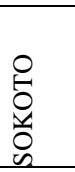 & $\begin{array}{l}\text { 己 } \\
\text { 己 } \\
\text { 己 }\end{array}$ & 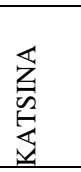 & 怘 & $\begin{array}{l}\text { 됫 } \\
5 \\
5 \\
0\end{array}$ \\
\hline \multicolumn{11}{|l|}{ YENAGOA } \\
\hline \multicolumn{11}{|l|}{$\mathrm{PH}$} \\
\hline \multicolumn{11}{|l|}{ OWERRI } \\
\hline ASABA & 123.8 & & & & & & & & & \\
\hline \multicolumn{11}{|l|}{ AWKA } \\
\hline \multicolumn{11}{|l|}{ UMUAHIA } \\
\hline \multicolumn{11}{|l|}{ UYO } \\
\hline \multicolumn{11}{|l|}{ CALABAR } \\
\hline \multicolumn{11}{|l|}{ ABAKALIKI } \\
\hline \multicolumn{11}{|l|}{ ENUGU } \\
\hline LOKOJA & 208.4 & & & & & & & & & \\
\hline \multicolumn{11}{|l|}{ ABUJA } \\
\hline \multicolumn{11}{|l|}{ LAFIA } \\
\hline \multicolumn{11}{|l|}{ MAKURDI } \\
\hline \multicolumn{11}{|l|}{ JALINGO } \\
\hline \multicolumn{11}{|l|}{ YOLA } \\
\hline \multicolumn{11}{|l|}{ MAIDUGURY } \\
\hline DAMATURU & & & & & & & & & & 285 \\
\hline \multicolumn{11}{|l|}{ GOMBE } \\
\hline BAUCHI & & & & & & & & & 232.2 & \\
\hline \multicolumn{11}{|l|}{ JOS } \\
\hline KADUNA & & & & & & & 199.8 & & 282.7 & \\
\hline MINNA & & & & & 349.2 & & & & & \\
\hline ILORIN & & & & 141.1 & 338.8 & & & & & \\
\hline OSHOGBO & & & & 82.6 & & & & & & \\
\hline \multicolumn{11}{|l|}{ ADOEKITI } \\
\hline AKURE & 113.5 & & & & & & & & & \\
\hline BENIN CITY & & 253.6 & & & & & & & & \\
\hline IKEJA & 253.6 & & 64.2 & 110.9 & & & & & & \\
\hline ABEOKUTA & & 67.9 & & 64.2 & & & & & & \\
\hline IBADAN & & 110.9 & 67.9 & & & & & & & \\
\hline BERIN KEBI & & & & & & 219.6 & & & & \\
\hline SOKOTO & & & & & 219.6 & & 185.8 & 361.9 & & \\
\hline$\overline{\text { GUSAU }}$ & & & & & & 185.8 & & 91.2 & & \\
\hline KATSINA & & & & & & 361.9 & 91.2 & & 113.9 & 209.3 \\
\hline KANO & & & & & & & & 113.9 & & 95.6 \\
\hline DUTSE & & & & & & & & 209.3 & 95.6 & \\
\hline
\end{tabular}

\section{Result}

The result of applying the Prim's algorithm to the network in figure 1 is as shown in figure 2. From the Minimum Spanning Tree in fig. 2 a total distance of $5,128.5 \mathrm{~km}$ is obtained.

\section{Minimum Spanning Tree Network of city to City Distance in Nigeria.}

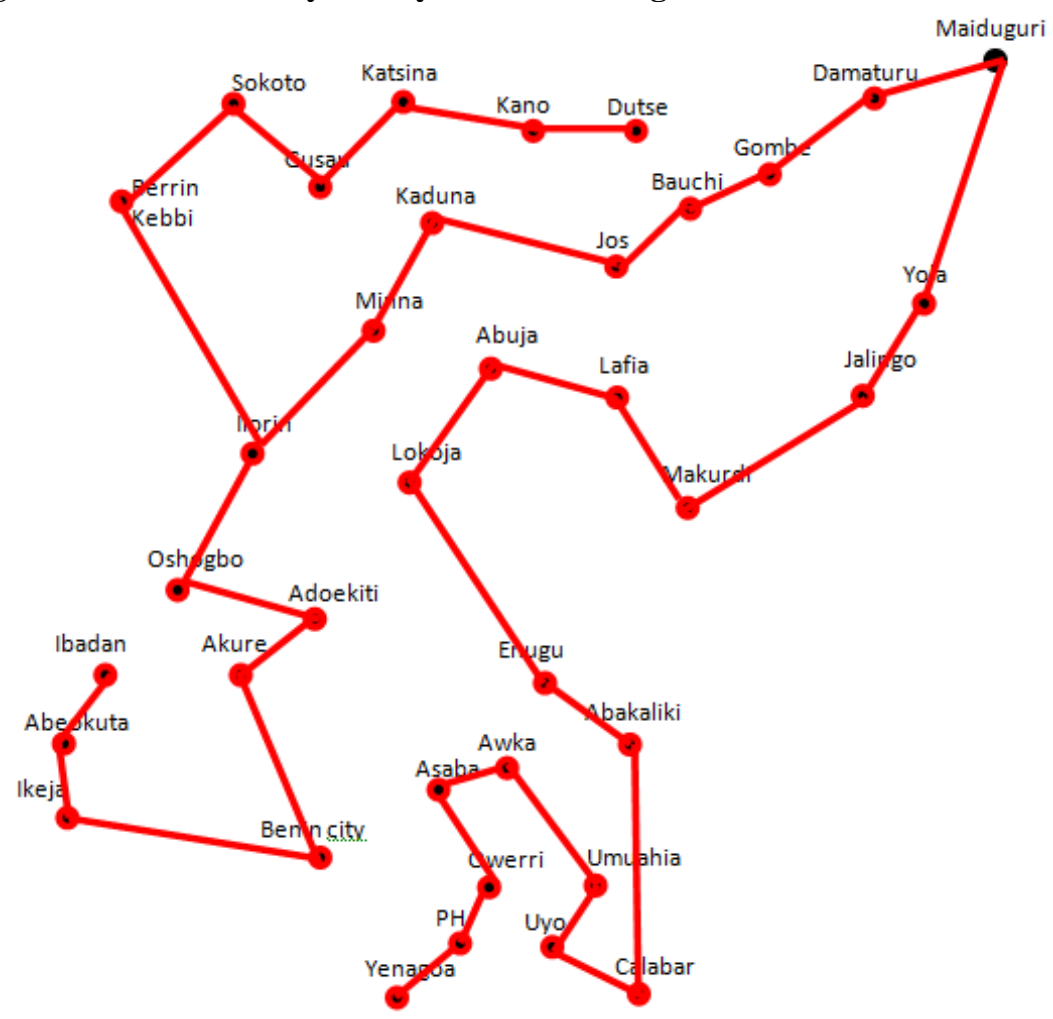

Fig.2

IV. Conclusion

The optimal road network so constructed can be used for various purposes in Nigeria, for example, designing of telecommunication network, transportation network, network of high - voltage electrical power transmission lines, network of gas and petroleum pipelines, etc. Although most of these networks have been in existence in Nigeria, the network design in this work would be needful in other applications yet to be considered. 


\section{References}

[1]. Agarwal,D.(2010).KruskalAlgorithm.Technicalcollection. http://sites.google.com/site/mytechnicalcollection/algoritms/graphs/minimumspanning- tree/kruskal's algorithm.

[2]. Ahuja, R. K., Magnantic, L. T., Orlin, J. B. and Reddy, R. M. (1992). Applications for NetworkOptimization. http://dspace.mit.edu/bitstream/handle/1721.1/5097/OR-3? Sequence=1.

[3]. Brimberg, J. N., Mladenovic, H., Pierre, L. K., and Breton, M. (2003). An oil pipeline design problem. Operations Research, Vol. 51, No. 2, Pp228 - 239. http://www.jstor.org/stable/4132402?Origin=JSTORpdf

[4]. Dippon, M. C. and Kenneth, E. T. (1999). The cost of local Telecommunication Network. A comparison of Minimum Spanning Tree and the HAI model. National Economic Research Associates, Enthropy Estimation Workshop.

[5]. Donkor, E. K., Amponsah, S. K. and Darkwa, F. K. (2010). Optimal connection for theWest African Gas Pipeline Project. http://Maxellsci.com/print/rjaset/v3-67-73pdf.

[6]. Dott,D.R.(1997).OptimalNetworkDesignforNaturalGaPipelines.UniversityofCalgary,Alberta.http://dspace.ucalgary.ca/bitstream/1880/26 992/1/31387Dott.pdf.

[7]. Mares, M. (2008). The Saga of Minimum Spanning Trees. Department of Applied Mathematics, Charles University, Czech Republic. http://www.sciencedirect.com.

[8]. Nahla, M. A. I. (2011). Optimal Spanning Trees. African Institute for Mathematical Science.http://www/users.aims.ac.za/nahla/nahla.pdf

[9]. Rothfard, B and Frank, K. (1970). Optimal design of offshore natural gas pipeline systems. Operations Research, Vol. 1, No. 6, Pp 992 1002. http://citeseerx.ist.psu.edu/viewdoc/download?doi=10.1.1.113

[10]. Seth, P and Vijaya, R. (2002). An Optimal Minimum Spanning Tree Algorithm. Journal of ACM, Vol. 49,

No. 1. Pp $16-33$

[11]. Taha, H. A. (2006). Operations Research. An introduction ( $7^{\text {th }}$ edition), Pearson Prentice Hall of India, New Delhi.

[12]. Wikipedia (2008). Transpport.http//en.wikipedia.org/wiki/pipeline transport.

[13]. Wiston, W. L. (2004). Operations Research; Applications and Algorithms ( $4^{\text {th }}$ edition), Brooks/Cole- Thomas Learning, Belmont. 